\title{
Non-equilibrium thermionic electron emission for metals at high temperatures
}

\author{
J.L. Domenech-Garret. $*$ S.P. Tierno, and L. Conde \\ Departamento de Física Aplicada, E.T.S.I. Aeronáutica y del Espacio. \\ Univ. Politécnica de Madrid, 28040 Madrid, Spain.
}

(Dated: August 23, 2018)

\begin{abstract}
The stationary thermionic electron emission currents from heated metals are compared against an analytical expression derived using a non equilibrium quantum Kappa energy distribution for the electrons. This later depends on the temperature decreasing parameter $\kappa(T)$ which can be estimated from the raw experimental data and characterizes the departure of the electron energy spectrum from the equilibrium Fermi-Dirac statistics. The calculations accurately predict the measured thermionic emission currents for both high and moderate temperature ranges. The Richardson-Dushman law governs the electron emission for large values of Kappa or equivalently, for moderate metal temperatures. The high energy tail in the electron energy distribution function which develops at higher temperatures or lower Kappa parameters, increases the emission currents well over the predictions of the classical expression. This analysis also permits the quantitative estimation of the departure of the metal electrons from the equilibrium Fermi-Dirac statistics.

PACS numbers: $05.90 .+\mathrm{m}$ 05.30.Fk 79.40.+z
\end{abstract}

\section{INTRODUCTION}

The thermionic electron emission from metals at high temperatures is today on the basis of countless technical applications. Different materials are currently used as hot cathodes to produce beams of negatively charged particles in electron guns, plasma sources or microwave devices such as klystrons or traveling wave tubes [1], and vacuum thermionic energy conversion devices [2].

The stationary flow of thermionic electrons from the metal surface is currently calculated by the classical Richardson-Dushman (RD) equation. This model considers the thermal equilibrium between the electron gas and the metal lattice and makes use of the Fermi-Dirac distribution for the electron energy spectrum [3]. The $\mathrm{RD}$ thermionic electron current density $J_{R D}(T)$ essentially relies on the work function $W_{f}$ of the metal and its temperature $T$, disregarding other important factors, such as the geometry [4, 5] of the emitting surface or its physical state [6].

However, the electron energy distribution in metals at high temperatures frequently differs from the FermiDirac statistics. The stationary RD electron emission regime represents the final step after different energy thermalization processes with shorter time scales [5, 79]. This time dependent decay towards the stationary thermal equilibrium have been studied by exposing the metal surfaces to ultrashort laser pulses. The subsequent evolution of the energy spectrum is later monitored to determine the electron energy relaxation rates [7, 9, 10].

The average electron energy relaxes fast, within the femtosecond time scale by collisions between electrons. Therefore, the electron $T_{e}$ and metal $T$ temperatures differ within these short times scales. The thermalization

*Electronic address: domenech.garret@upm.es between the electron gas and the metal lattice requires of few tens of picoseconds due to the larger mass of phonons [5, 7 -9]. The RD regime is considered to provide an adequate description of the electron emission for long characteristic times when the electron gas and the metal lattice reach the thermal coupling $T=T_{e}$ [8, 9, 11]. Nevertheless, this pure RD thermionic emission regime of electrons has not been observed because the energy of the laser pulse lies over damage thresholds of most metals 7].

This physical description relies on the implicit assumption of the long time scale relaxation to a thermal equilibrium where the Fermi-Dirac statistics describes the electron energy spectrum [7, 9]. This might be not always the case, even under an efficient energy transfer between the electron gas and metal lattice. For high temperatures the metal might remain out of equilibrium because additional energy exchange processes take place, such as the intense emission of electromagnetic radiation, the development strong surface thermal gradients or the nonuniform electron emission. The high energy interactions between the electrons and phonons couple the electron gas with the overheated metal lattice, producing groups of fast electrons [5, 9]. Therefore, even for long time scales, the electron energy spectrum might differ from the Fermi-Dirac energy distribution in hot metals.

Consequently, the equilibrium Fermi-Dirac statistics needs to be replaced to account for these high energy electron groups. The usual equilibrium statistical physics cannot cope with these non-conventional electron energy distribution functions previously proposed in different fields [12]. Namely, the derivation of a generalized Planck radiation law 13], the description of high temperature Fermi gases [14] or the out of equilibrium warm dense matter [15].

Recently, an analytical expression was derived for the thermionic current density $J_{\kappa}(T)$, using a modified Kappa energy distribution for the electrons [16]. This 


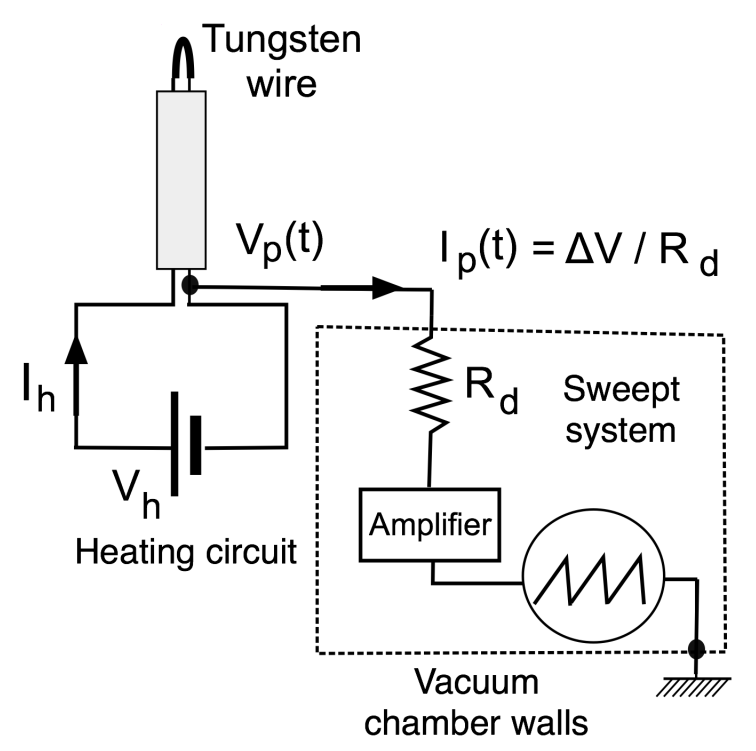

Figure 1: The diagram of experimental setup where, $V_{h}$, is the voltage drop across the tungsten wire heated by the DC current, $I_{h}$. The dotted box represents the scheme of the sweep bias circuit which applies an amplified sawtooth waveform $V_{p}(t)$ to the heated wire and measure the emission current, $I_{p}(t)$, as the voltage drop, $\Delta V$, across a precision resistor, $R_{d}$.

formula corresponds to the high energy, non equilibrium Fermi-Dirac distribution [17, 18]. The index $\kappa$ of this distribution accounts for the high energy tail in the electron energy spectrum, as well as the Kappa deformed statistics previously proposed [19].

In this paper the measurements of the stationary thermionic emission currents from metals are found in good agreement with the theoretical predictions of Ref. [16]. The RD expression underestimates the experimental emission currents for high metal temperatures, whereas $J_{R D}(T)$ and $J_{\kappa}(T)$ agree for moderate values.

As we shall see, the electron energy distribution might be approximated using the temperature dependent $\kappa(T)$ index of this statistics, which could be determined from the experimental data. This $J_{\kappa}(T)$ accounts for the contribution of high energy electrons in the thermionic electron emission.

Additionally, it might be employed as a quantitative estimation of the the departure from the thermal equilibrium described by the Fermi-Dirac statistics which is recovered in the limit of large $\kappa$. To our best knowledge, this present analysis is one of the few cases where the predictions of a non equilibrium quantum Kappa distribution are directly compared against raw experimental data.

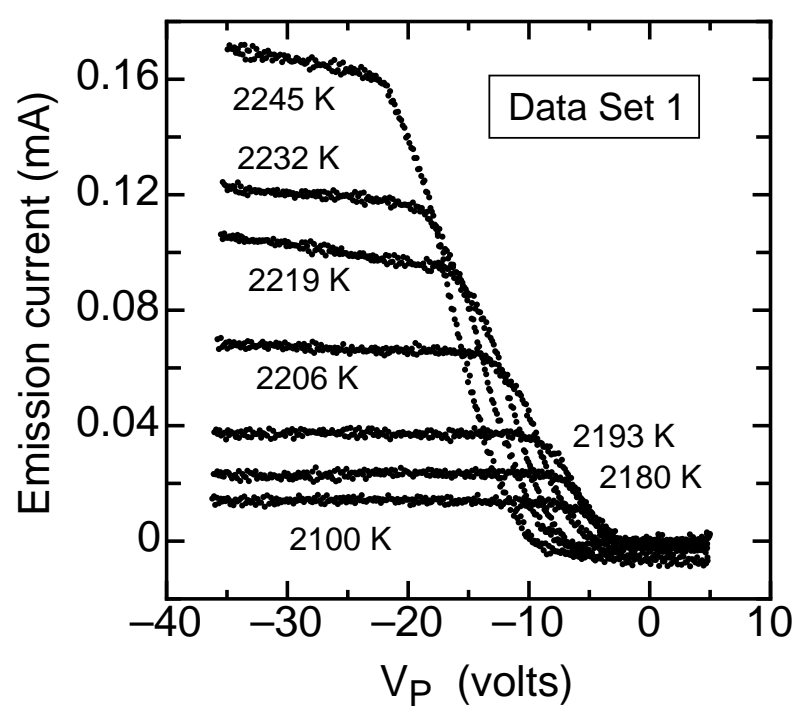

Figure 2: The electron emission currents $I_{e}(T)$ as a function of the bias potential $V_{p}$ for different metal temperatures.

\section{THE NON EQUILIBRIUM ELECTRON EMISSION MODEL}

The stationary thermionic electron current from metals at high temperatures could be calculated using the stationary, quantum non-equilibrium Kappa statistics [16],

$$
f_{\kappa}\left(T_{e}, E\right)=C_{\kappa}\left(T_{e}\right)\left(1+\frac{E-\epsilon_{F}}{k_{B} T_{\kappa}}\right)^{-(\kappa+1)}
$$

Here $k_{B}$ is the Boltzmann constant and $E$ is the kinetic energy of electrons of mass $m_{e}$, which is evaluated with respect to the Fermi level $\epsilon_{F}$. The other parameters are $\gamma_{1}=\epsilon_{F} / k_{B} T_{e}$ and $T_{\kappa}=\left(\kappa-3 / 2+\gamma_{1}\right) T_{e}$. Eq. (2.1) is the approximation for high energies of the generalized non equilibrium quantum statistics of Refs. [17] and [18]. The normalization factor $C_{\kappa}\left(T_{e}\right)$ in Eq. (2.1) scales to the metal electron density $n_{e o}$ as,

$$
\begin{array}{r}
C_{\kappa}\left(T_{e}\right)=n_{e o} \frac{\Gamma(\kappa+1)}{\Gamma\left(\kappa-\frac{1}{2}\right)} \times \\
\times\left(\frac{m_{e}}{(\kappa-3 / 2) 2 \pi k_{B} T_{e}}\right)^{3 / 2}\left(\frac{\kappa-3 / 2}{\kappa-3 / 2+\gamma_{1}}\right)^{\kappa+1}
\end{array}
$$

The low values of $\kappa$ in Eq. (2.1) account for a large high energy tail in the electron energy spectrum, whereas large indexes $\kappa$ recover the classical Fermi-Dirac statistics [16].

For long time scales, well over the microsecond range equal temperatures $T_{e}=T$ are considered for the electron gas and the metal lattice [7, 9, 11]. The average electron thermal energy proportional to $k_{B} T_{e}$ is always much 
lower than the work function, $W_{f}$. In these conditions the analytical expression for the stationary thermionic electron current density $J_{\kappa}(T)$ deduced from Eq. (2.1) reads [16],

$$
\begin{array}{r}
J_{\kappa}(T)=e C_{\kappa}(T) \frac{\pi k_{B}^{2} T^{2}}{m_{e}^{2}} \times \\
\frac{\left(2 \kappa-3+2 \gamma_{1}\right)^{2}}{2 \kappa(\kappa-1)} \times\left(1+\frac{W_{f}}{k_{B} T_{\kappa}}\right)^{-\kappa+1}
\end{array}
$$

For low $\kappa$ the thermionic electron fluxes predicted by Eq. (2.2) are higher than those calculated using the classical Richardson-Dushmann expression $J_{R D}(T)$, which is also recovered in the opposite limit of large $\kappa$ [16].

The contribution in $J_{\kappa}(T)$ of the high energy tail of the electron energy distribution function increases with the metal temperature. This effect is incorporated by means of a temperature dependent index $\kappa(T)$ in either $f_{\kappa}(T, E)$ and $J_{\kappa}(T)$.

\section{EXPERIMENTS}

The predictions of Eq. (2.2) were checked against the measurements of the thermionic electron currents $I_{e}\left(T, V_{p}\right)$ from a DC heated tungsten and in Fig. 1 is depicted a simplified scheme of the experimental setup. The loop shaped wire with a typical length of $22 \mathrm{~mm}$ and $0.08 \mathrm{~mm}$ in diameter was placed at the end of a electrically insulated ceramic shaft. The emitting wire was approximately located at the center of a cylindrical vacuum chamber of $0.8 \mathrm{~m}$ in length and $0.4 \mathrm{~m}$ in diameter evacuated down to typical pressures below $10^{-5} \mathrm{mB}$ of Argon. The elastic collisions between electrons and neutral is the dominant collisional process. According to [20] the cross sections are $9-20 \times 10^{-20} \mathrm{~m}^{2}$, which gives typical mean free paths between 20 and $44 \mathrm{~m}$, much larger than our experimental arrangement. This fact excludes the production of additional charged particles because the ionizing and elastic collisions between electron and neutrals are negligible.

The tungsten wire was heated up to thermionic electron emission by a DC currents of $I_{h} \simeq 0.8-1.0 \mathrm{~A}$ with voltages $V_{h} \simeq 2-3 \mathrm{~V}$ and electrically biased with respect to the grounded walls of the vacuum tank. For these thin tungsten filaments the temperature gradients along the hot wire could be neglected and energy power losses are mainly caused by radiation emission. Therefore, the temperatures were determined using a well known expression relating the DC heating current along the wire with $T$, which is regarded essentially uniform [21 23].

The sweep system impress an amplified time dependent sawtooth signal, $V_{p}(t)$, with a repetition pulse of 2 $\mathrm{kHz}$ to one leg of the heated tungsten wire [24]. As shows Fig. 1 the emitted thermionic electron current $I_{e}\left(T, V_{p}\right)$ is obtained from the current, $I_{p}(t)$, measured as the voltage drop, $\Delta V$, across the precision resistance, $R_{d}$.

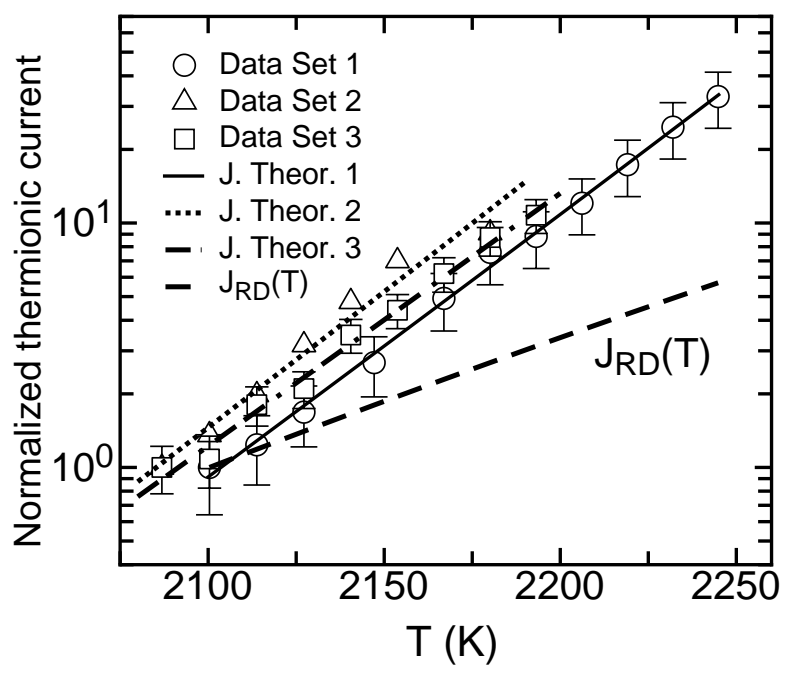

Figure 3: Comparison between the experimental data $I_{s}(T) / I_{s}\left(T_{o}\right)$ and the normalized thermionic current densities $j_{\kappa}(T)=J_{\kappa}(T) / J_{\kappa_{m}}\left(T_{o}\right)$ (labeled as J. Theor.) by Eq. (2.2) using the fitting $\kappa(T)$ of Fig. 4 The dashed line represents the predictions of the normalized Richardson-Dushmann equation $j_{R D}(T)$ as a function of the metal temperature.

The typical electron emission currents $I_{e}\left(T, V_{p}\right)$ are represented in Fig. 2 for different metal temperatures $T$ as a function of the bias potential $V_{P}$ of the wire. For negative bias potentials $V_{p}$ the thermionic electrons are emitted from the wire towards the vacuum chamber walls, whereas no electron current is observed for positive potentials.

The lower currents in Fig. 2 were of tens of $\mu \mathrm{A}$ corresponding to $T<2100 \mathrm{~K}$ that raised up to the $0.1-0.2 \mathrm{~mA}$ range for metal temperatures over $2200 \mathrm{~K}$. For low positive potentials where the electron emission becomes negligible, $I_{e}\left(T, V_{p}\right)$ reaches a flat negative current for voltages over a threshold of about 5 volts. The thermionic electron emission takes place for negative bias potentials and large slopes in the range -5 to -20 volts correspond to the space charge effects around the wire. These are outweighed over a typical threshold bias potential of the wire corresponding to the knee of $I_{e}\left(T, V_{p}\right)$ where the flat electron emission current becomes weakly dependent on the bias potential.

Then, the flat saturation currents $I_{s}(T) \simeq I_{e}\left(T, V_{p}\right)$ for $V_{p} \leq-20$ volts past the knee of $I_{e}\left(T, V_{p}\right)$, measure the maximum emitted electron thermionic current. The moderate bias voltages $V_{p}$ involved in Fig. 2 exclude from this pure thermionic regime the thermofield or FowlerNordheim or field emission modes [25, 26].

\section{EXPERIMENTAL DATA ANALYSIS}

The theoretical predictions of Eq. (2.2) are compared with the measurements of $I_{s}(T)$ in Fig. 3. For low metal 
temperatures (typically below $2000 \mathrm{~K}$ ) both, the RD expression and Eq. (2.2) give similar thermionic electron emission currents. However, the increment in the metal temperature leads to important differences.

Additionally, the values of index $\kappa(T)$ of $f_{\kappa}(T, E)$ could be evaluated from the maximum thermionic electron emission currents $I_{s}(T)$ and are represented in Fig. 4 The low temperature limit corresponds in Fig. 3 to large values of the index $\kappa(T)$ where $f_{\kappa}(T, E)$ becomes similar to the equilibrium Fermi-Dirac statistics. The decreasing $\kappa(T)$ index characterizes the $f_{\kappa}(T, E)$ for growing metal temperatures.

The following procedure was used in Figs. 3 and 4 to avoid the need of the accurate determination of the electron emitting metal surface, as well as the particular value of the Richardson constant for the metallic samples.

First, we obtain the parameter $\kappa_{m}$ for the lowest metal temperatures $T_{o} \simeq 2100 \mathrm{~K}$ in Fig. 2, which corresponds to the Richardson-Dushman electron emission regime. From Eq. (2.2) and the classical RD expression we obtain $k_{m}$ as the root of a nonlinear equation by setting,

$$
\frac{J_{\kappa_{m}}\left(T_{o}\right)}{J_{R D}\left(T_{o}\right)}=1
$$

This permits to evaluate the lower current density $J_{\kappa_{m}}\left(T_{o}\right)$ using Eq. (2.2) which corresponds to the lower measured thermionic current $I_{s}\left(T_{o}\right)$ of Fig. 2, The maximum values is about $k_{m} \simeq 25.6-26.0$ in Fig. 4 for the three sets of experimental data.

Next, using the maximum thermionic emission currents $I_{s}(T)$ for the metal temperature $T$ the ratios $I_{s}(T) / I_{s}\left(T_{o}\right)$ are therefore,

$$
\frac{I_{s}(T)}{I_{s}\left(T_{o}\right)}=\frac{J_{\kappa}(T)}{J_{\kappa_{m}}\left(T_{o}\right)}=j_{\kappa}(T)
$$

Using Eq. (2.2) the values $\kappa(T)<\kappa_{m}$ for increasing temperatures $T>T_{o}$ could be again evaluated as the roots of a nonlinear equation.

Finally, the temperature decreasing values of $\kappa(T)$ of Fig. 4 could be approximated by $\kappa(T)=a-b T$ by means of a least-squares fitting and these empirical expressions are later introduced in Eq. (2.2). This procedure allows to compare its theoretical predictions with the experimental ratios $I_{s}(T) / I_{s}\left(T_{o}\right)$. The normalized curves $j_{\kappa}(T)$ along with the experimental ratios $I_{s}(T) / I_{s}\left(T_{o}\right)$ are represented in Fig. 4 as well as,

$$
j_{R D}(T)=\frac{J_{R D}(T)}{J_{R D}\left(T_{o}\right)}
$$

which it were calculated by using the classical Richardson-Dushmann equation.

\section{CONCLUSIONS}

The classical Richardson-Dushman equation based on the equilibrium Fermi-Dirac statistics adequately de-

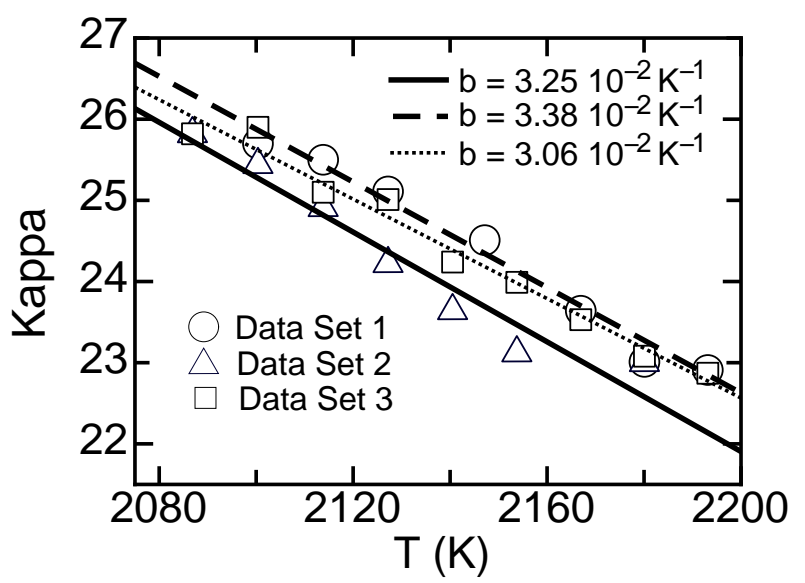

Figure 4: The parameter $\kappa(T)$ corresponding to the experimental data fitting, using the least-squares method, of the three data sets of Fig. (3) to $\kappa(T)=a-b T$, where $T$ is the metal temperature.

scribes the thermionic electron emission for low and moderate metal temperatures. However, additional energy exchange processes bring the metal lattice far form the thermal equilibrium at higher temperatures. Thus, a fraction the lattice energy is also transferred to the electron gas for long time scales. In these conditions, the electron energy spectrum differs from the Fermi-Dirac statistics and could be approximated by the effective, non equilibrium quantum Kappa distribution of Eq. (2.1). This later recovers the Fermi-Dirac statistics for low temperatures or equivalently, for large values of the $\kappa(T)$ index in $f_{\kappa}(T, E)$.

The empirical determination of the index $\kappa(T)$ from the stationary thermionic electron emission currents $I_{s}(T)$ for different metal temperatures was discussed in Sec. IV. The maximum value $\kappa_{m}$ for the lower temperature in Fig. 4 corresponds to the RD regime and the decreasing function $\kappa(T)$ quantitatively characterizes the departure of the electron energy spectrum from the Fermi-Dirac statistics [16]. This transition rate is governed by the slope $b$ in the empirical fit to to $\kappa(T)=$ $a-b T$ of Fig. 4 which also characterize the increasing high energy tail in $f_{\kappa}(T, E)$ with the metal temperature.

Fig. 3 shows the good agreement between the experimental data and the thermionic electron emissions currents $J_{\kappa}(T)$ calculated using $f_{\kappa}(T, E)$ for the electron energy spectrum and the empirical expressions for $\kappa(T)$. Additionally, the measurements of Fig. 3 show the increase of the thermionic current by orders of magnitude over RD law predictions for high metal temperatures. These increments are caused by the departure for the thermal equilibrium of both the metal lattice and the electron gas not contemplated by the equilibrium FermiDirac statistics employed in the derivation of the classical RD equation.

This high thermionic electron emission regime is explained by the contribution of high energy electron 
groups in the energy spectrum of the metal electrons. Therefore, Eq. (2.2) extends the predictions of the classical Richardson-Dushman equation to higher metal temperature and permits to make us of $\kappa(T)$ to approximate the stationary energy distribution function of electrons in hot metals.

Finally, regarding the applicability of these results, cathodes with higher thermionic electron emission currents would led to improved characteristics in electron tube devices. Larger electron currents lead to greater amplification factors in traveling wave tubes or a better coupling with the microwave input signal in klystrons.

\section{Acknowledgments}

The authors are grateful to the MICINN funding through Grant ESP2013-41078-R and S.P. Tierno acknowledges the financial support through FPU program from the Spanish Ministry of Education.
[1] J.J. Carr, Microwave $\&$ Wireless Communications Technology (Elsevier, New York, 1996), Chap. 11.

[2] J.R. Smith, J. Appl. Phys. 114, 164514 (2013).

[3] R.B. Lindsay, Introduction to Physical Statistics (Wiley, New York, 1962) Chap. 11.

[4] J.K. Wysocki, Phys. Rev. B 28, 834 (1983).

[5] D.M. Riff, X.Y. Wang, M.C. Downer, D.L. Fisher, T. Tajima, J.L. Erskine, and R.M. More. J. Opt. Soc. Am. B. 10, 1424 (1993).

[6] Y.U. Paderno, A.A. Taran, D.A. Voronovich, V.N. Paderno and V.B. Filipov, Functional Materials 15, 63 (2008).

[7] G. Ferrini, F. Banfi, C. Giannetti and F. Parmigian, Nuc. Inst. Meth. Phys. Res. A, 601, 123 (2009).

[8] W. Wendelen, B.Y. Mueller, D. Autrique, B. Rethfeld and A. Bogaerts, J. Appl. Phys. 111, 113110 (2012).

[9] B. Rethfeld, A. Kaiser, M. Vicanek and G. Simon. Phys. Rev. B, 65, 214303 (2002).

[10] S.G. Bezhanov, A.P. Kanavin and S.A. Uryupin, Quantum Electronics 42, 447 (2012).

[11] P.B. Corkum, F. Brunel and N.K. Sherman, T. Srinivasan-Rao, Phys. Rev. Lett. 61, 2886 (1988).

[12] K. Ourabah, L. Ait Gougam and M. Tribeche, Phys. Rev. E, 91, 012133 (2015).

[13] K. Ourabah and M. Tribeche, Phys. Rev. E, 89, 062130
(2014)

[14] A. Algin and M. Senay, Phys. Rev. E 85, 041123 (2012).

[15] J. Clérouin, G. Robert, P. Arnault, C. Ticknor, J.D. Kress and L.A. Collins, Phys. Rev. E, 91, 011101(R) (2015).

[16] J.L. Domenech-Garret, S.P. Tierno and L. Conde, Eur. Phys. J. B, 86, 382 (2013).

[17] G. Livadiotis, D.J. McComas, Astrophs. J. 741:88 (2011).

[18] R.A. Treumann, Europhys. Lett., 48, 8 (1999).

[19] G. Kaniadakis, Physica A 296, 405 (2001).

[20] G.G. Raju, IEEE Trans. on Dielectrics and Electrical Insulation 11(4), 649 (2004), Figure 1 Page 654.

[21] I. Langmuir, Phys. Rev. 7, 302 (1916).

[22] S. Halas and T. Durakiewicz, Vacuum, 49, (4) pp. 331336 (1998).

[23] S.P. Tierno, J.L. Domenech-Garret, J.M. Donoso, D. Jennewein, G. Herdrich, S. Fasoulas, and L. Conde, IEEE Trans. Plasma Sci. 41, 695 (2013).

[24] O. Troll, L. Conde, E. Criado, J.M. Donoso, and G. Herdrich. Contrib. to Plasma Phys. 50(9), 819 (2010).

[25] E.L. Murphy and R.H. Good, Phys. Rev. 102, (6) 1464 (1956).

[26] S. Coulombe and J.L. Meunier, J. Phys. D: Appl. Phys. 30776 (1997). 